\title{
Rigorous Solutions of Large-Scale Scattering Problems Discretized with Hundreds of Millions of Unknowns
}

\author{
L. Gürel ${ }^{*, \dagger}$
}

\begin{abstract}
We present fast and accurate solutions of large-scale scattering problems using a parallel implementation of the multilevel fast multipole algorithm (MLFMA). By employing a hierarchical partitioning strategy, MLFMA can be parallelized efficiently on distributed-memory architectures. This way, it becomes possible to solve very large problems discretized with hundreds of millions of unknowns. Effectiveness of the developed simulation environment is demonstrated on various scattering problems involving canonical and complicated objects.
\end{abstract}

\section{INTRODUCTION}

The multilevel fast multipole algorithm (MLFMA) is a powerful method for the fast and efficient solution of electromagnetics problems discretized with large numbers of unknowns [1]. Nevertheless, accurate solutions of real-life problems often require discretizations with millions of unknowns. In order to handle such extremely-large-scale problems, MLFMA must be parallelized on a cluster of computers [2]-[5]. Unfortunately, efficient parallelization of MLFMA is not trivial due to the complicated structure of this algorithm. In this paper, we present an overview of our efforts to develop parallel implementations of MLFMA for the solution of very large scattering problems discretized with hundreds of millions. We demonstrate the feasibility of accurately solving large-scale problems on relatively inexpensive computing platforms without resorting to approximation techniques.

\section{SIMULATION ENVIRONMENT}

In this section, we summarize the major components of our simulation environment.

\subsection{Formulation and Discretization}

Problems considered in this paper involve closed conducting surfaces, which can be formulated with the combined-field integral equation (CFIE). CFIE

\footnotetext{
*Department of Electrical and Electronics Engineering, Bilkent University, TR-06800, Bilkent, Ankara, Turkey.

${ }^{\dagger}$ Computational Electromagnetics Research Center (BiLCEM), Bilkent University, TR-06800, Bilkent, Ankara, Turkey.

e-mail: \{lgurel, ergul\}@ee.bilkent.edu.tr

tel.: +90 312 2905750, fax: +90312 2905755 .
}

is the convex combination of the electric-field integral equation (EFIE) and the magnetic-field integral equation (MFIE), i.e., CFIE $=\alpha \mathrm{EFIE}+(1-$ $\alpha$ )MFIE. The coupling parameter $\alpha$ is set to 0.2 for optimal iterative convergence. CFIE is discretized with the Rao-Wilton-Glisson functions defined on planar triangles smaller than $\lambda / 10$, where $\lambda$ is the wavelength.

\subsection{Iterative Solutions}

Iterative solutions are performed by using the biconjugate-gradient-stabilized algorithm, which is known to provide rapid solutions for CFIE. In all solutions, the relative residual error for the iterative convergence is set to $10^{-3}$. Solutions are further accelerated by employing block-diagonal preconditioners that are constructed from the self interactions of the lowest-level subdomains in MLFMA.

\section{$2.3 \quad$ Near-Field Interactions}

In MLFMA, there are $\mathcal{O}(N)$ near-field interactions, which are calculated directly and stored in memory to be used multiple times during iterations. We use singularity-extraction techniques, adaptive integration methods, and Gaussian quadratures in order to calculate near-field interactions accurately and efficiently. Typically, near-field interactions are calculated with a maximum of $0.5 \%$ error.

\subsection{Far-Field Interactions}

For each problem, a tree structure is constructed by placing the object in a cubic box and recursively dividing the computational domain into subdomains, until the box size is about $0.25 \lambda$. Then, far-field interactions are calculated in three stages, namely, aggregation, translation, and disaggregation. Truncation numbers, sampling rates for radiated and incoming fields, and numbers of interpolation/anterpolation points are selected carefully such that far-field interactions are calculated with a maximum of $1 \%$ error.

\subsection{Parallelization}

In order to solve large-scale problems, MLFMA must be parallelized on a cluster of computers, but 
this is not trivial due to the complicated structure of this algorithm. Specifically, distributing the multilevel tree structure among processors has been a major bottleneck for the efficient parallelization of MLFMA. Recently, we proposed a hierarchical parallelization strategy, which is based on the optimal partitioning at each level of MLFMA [5]. In this strategy, both subdomains and their samples are partitioned among processors by employing loadbalancing algorithms. The hierarchical strategy offers a higher parallelization efficiency than previous approaches, due to the improved load-balancing and the reduced number of communication events.

\subsection{Computing Platform}

Solutions presented in this paper are performed on a cluster of Intel Xeon Dunnington processors with $2.40 \mathrm{GHz}$ clock rate. The cluster consists of $16 \mathrm{com}-$ puting nodes, and each node has 48 GB of memory and multiple processors. For all solutions, we employ four cores per node (a total of 64 cores).

\section{RESULTS}

In this paper, we present solutions of scattering problems involving four metallic objects depicted in Figure 1, namely, a $1 \mathrm{~m} \times 3.5 \mathrm{~m} \times 0.1 \mathrm{~m}$ rectangular box, a wing-shaped object with sharp edges and corners, the NASA Almond of length $25.23 \mathrm{~cm}$, and the stealth airborne target Flamme with a maximum dimension of $0.6 \mathrm{~m}$.

\subsection{Rectangular Box}

The rectangular box is investigated at $75 \mathrm{GHz}$. At this frequency, the size of the box corresponds to $875 \lambda$ and its discretization with $\lambda / 10$ triangles leads to matrix equations involving $174,489,600$ unknowns. The box is illuminated by two plane waves propagating on the $x-z$ plane at $30^{\circ}$ and $60^{\circ}$ angles from the $z$ axis. The electric field is polarized in the $\theta$ direction. Two solutions are performed in a total of 13 hours. Figure 2 depicts the co-polar bistatic RCS (dBms) on the $x-z$ plane as a function of the bistatic angle $\theta$. In addition to the forwardscattering direction, RCS values make peaks at two reflection directions, i.e., at $150^{\circ}$ and $330^{\circ}$ for the $30^{\circ}$ illumination and at $120^{\circ}$ and $300^{\circ}$ for the $60^{\circ}$ illumination.

\subsection{Wing-Shaped Object}

The wing-shaped object with a maximum dimension of $1 \mathrm{~m}$ is investigated at $150 \mathrm{GHz}$. The object is discretized with $172,228,608$ unknowns, and it is illuminated by two plane waves propagating on the $x-y$ plane at $30^{\circ}$ and $60^{\circ}$ angles from the $x$ axis.
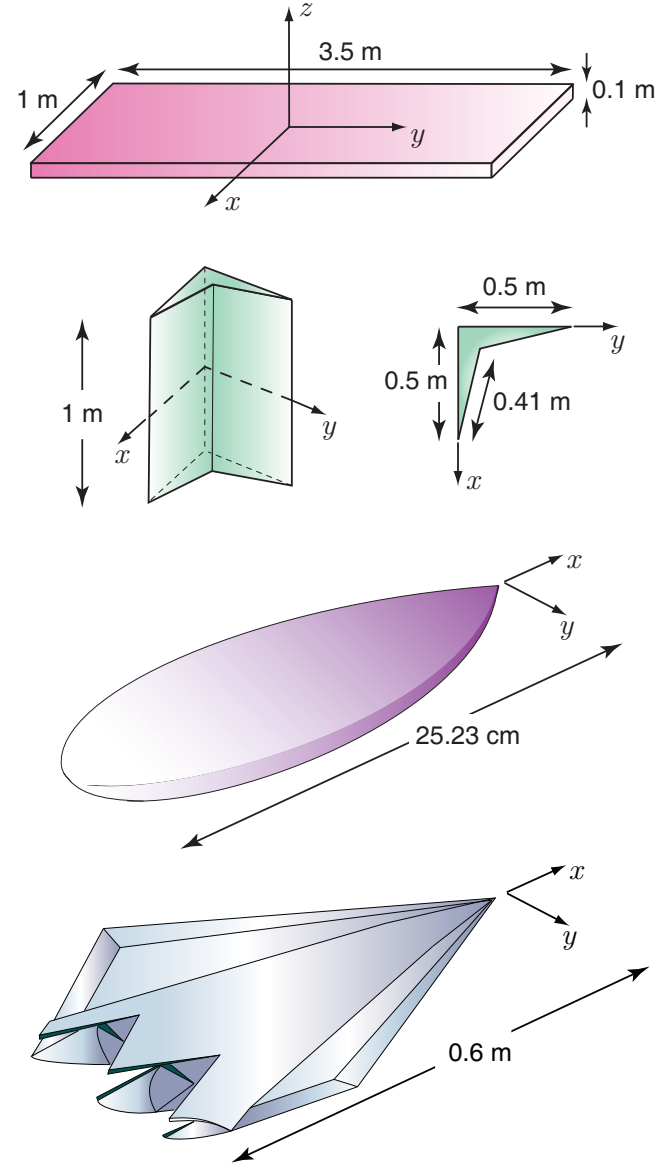

Figure 1: Large metallic objects, whose scattering problems are solved by the parallel MLFMA implementation.

The electric field is polarized in the $\phi$ direction. Two solutions are performed in a total of 15 hours. Figure 3 depicts the co-polar bistatic RCS (dBms) on the $x-y$ plane as a function of the bistatic angle $\phi$. We observe that RCS values of the wingshaped object make peaks at three directions due to reflections, in addition to the forward-scattering direction.

\subsection{NASA Almond}

The NASA Almond is discretized with 203,476,224 unknowns and investigated at $1.1 \mathrm{THz}$. The maximum dimension of the target corresponds to $915 \lambda$ at this frequency. The target is again illuminated by two plane waves propagating on the $x-y$ plane at $30^{\circ}$ and $60^{\circ}$ angles from the $x$ axis (from the nose). The electric field is polarized in the $\theta$ direction. Solutions are performed in a total of 22 hours. As depicted in Figure 4, the NASA Almond has a stealth ability with very low back-scattered RCS compared to the forward-scattered RCS. 

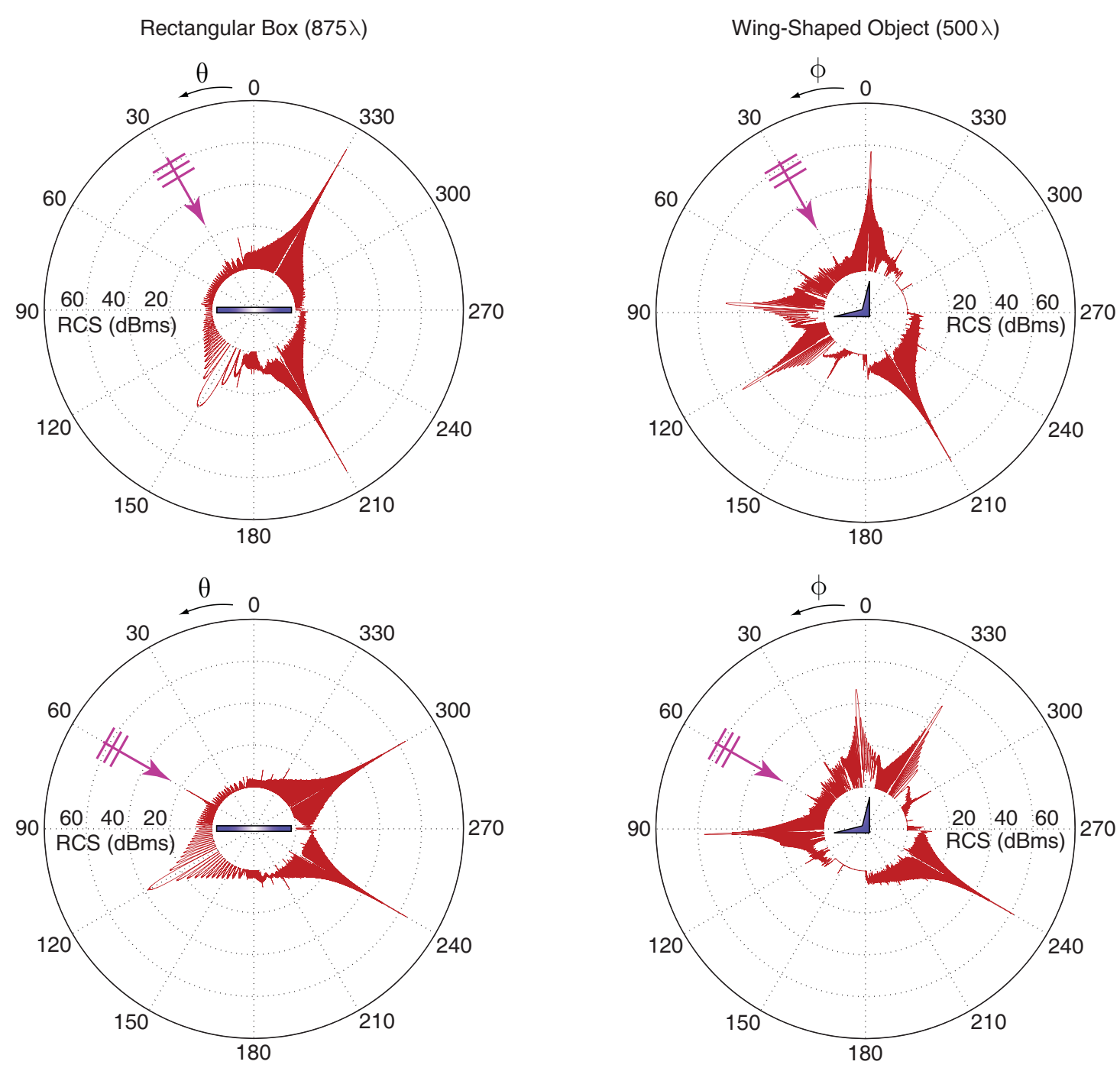

Figure 2: Co-polar bistatic RCS (dBms) of the rectangular box in Figure 1 at $75 \mathrm{GHz}$.

\subsection{Flamme}

Finally, the stealth airborne target Flamme is investigated at $440 \mathrm{GHz}$. The scaled size of the Flamme is $0.6 \mathrm{~m}$ and corresponds to $880 \lambda$ at this frequency. Discretization of the target with $\lambda / 10$ triangles leads to matrix equations involving 204,664,320 unknowns. Similar to the wing-shaped object and the NASA Almond, the Flamme is illuminated by two plane waves propagating on the $x-y$ plane at $30^{\circ}$ and $60^{\circ}$ angles from the $x$ axis with the electric field polarized in the $\theta$ direction. Solutions are performed in a total of 44 hours. Figure 5 shows that the back-scattered RCS of the Flamme is very low due to the stealth ability of this target, similar to the NASA Almond. In addition, depending on the illumination, we observe that the Flamme RCS ex-

Figure 3: Co-polar bistatic RCS (dBms) of the wing-shaped object in Figure 1 at $150 \mathrm{GHz}$.

hibits several peaks in various directions.

\section{CONCLUSION}

This paper presents rigorous solutions of scattering problems discretized with hundreds of millions of unknowns using a parallel implementation of MLFMA in order to demonstrate the feasibility of accurately solving large-scale problems on relatively inexpensive computing platforms without resorting to approximation techniques.

\section{Acknowledgments}

This work was supported by the Turkish Academy of Sciences in the framework of the Young Scientist Award Program (LG/TUBA-GEBIP/20021-12), by the Scientific and Technical Research 

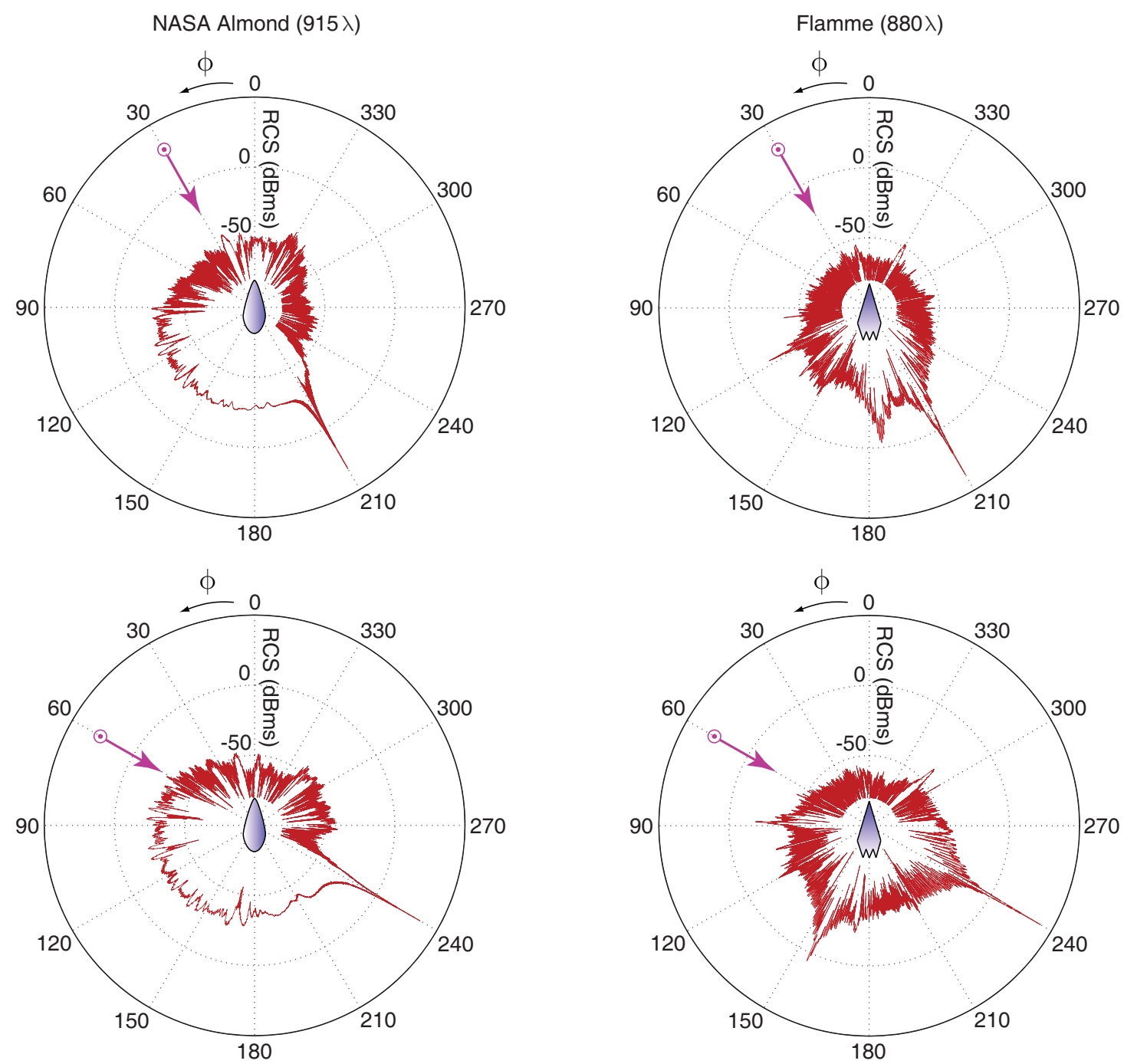

Figure 4: Co-polar bistatic RCS (dBms) of the NASA Almond in Figure 1 at $1.1 \mathrm{THz}$.

Council of Turkey (TUBITAK) under Research Grants 105E172 and 107E136, and by contracts from ASELSAN and SSM.

\section{References}

[1] J. Song, C.-C. Lu, and W. C. Chew, "Multilevel fast multipole algorithm for electromagnetic scattering by large complex objects," IEEE Trans. Antennas Propag., vol. 45, no. 10, pp. 1488-1493, Oct. 1997.

[2] S. Velamparambil and W. C. Chew, "Analysis and performance of a distributed memory multilevel fast multipole algorithm," IEEE Trans. Antennas Propag., vol. 53, no. 8, pp. 2719-2727, Aug. 2005.

Figure 5: Co-polar bistatic RCS (dBms) of the Flamme in Figure 1 at $440 \mathrm{GHz}$.

[3] Ö. Ergül and L. Gürel, "Efficient parallelization of the multilevel fast multipole algorithm for the solution of large-scale scattering problems," IEEE Trans. Antennas Propag., vol. 56, no. 8, pp. 2335-2345, Aug. 2008.

[4] J. Fostier and F. Olyslager, "An asynchronous parallel MLFMA for scattering at multiple dielectric objects," IEEE Trans. Antennas Propag., vol. 56, no. 8, pp. 2346-2355, Aug. 2008.

[5] Ö. Ergül and L. Gürel, "A hierarchical partitioning strategy for an efficient parallelization of the multilevel fast multipole algorithm," IEEE Trans. Antennas Propag., vol. 57, no. 6, pp. 1740-1750, Jun. 2009. 